\title{
Influence of crystal planes on corrosion behavior of tantalum: Experimental and first-principles study
}

\author{
Yuan Luo ${ }^{1}$, Mengran Bi ${ }^{1}$, Hongzhong Cai ${ }^{1, *}$, Changyi Hu ${ }^{l}$, Yan Wei ${ }^{l}$, Li Chen ${ }^{l}$, \\ Zhentao Yuan ${ }^{2, *}$, Xiao Wang ${ }^{2, *}$ \\ ${ }^{1}$ Kunming Institute of Precious Metals, Kunming, State Key Laboratory of Advanced Technologies \\ for Comprehensive Utilization of Platinum Metals, 650106, China; \\ ${ }^{2}$ City College, Kunming University of Science and Technology, Kunming, 650093, China; \\ *E-mail: chz@ipm.com.cn, kmust_welding@163.com, wang_xiao@kust.edu.cn
}

Received: 9 November 2021 / Accepted: 27 December 2022 / Published: 2 February 2022

In order to improve the corrosion resistance of tantalum coatings produced by chemical vapor deposition (hereinafter referred to as CVD Ta) in various industrial fields, the effect of the texture on the corrosion behavior was investigated. The microstructure and electrochemical properties of CVD Ta were examined by scanning electron microscopy (SEM), X-ray diffraction (XRD), and electrochemical analysis. The surface energy and work function of the Ta surface were investigated based on firstprinciples calculations, which were used for the simulations of polarization curves. The SEM analysis revealed that the coatings showed a microscopic pyramid structure. Furthermore, XRD analysis results showed that the coatings mainly exhibited (110), (200), and (211) planes. Electrochemical tests showed that the coatings with a preferential orientation along the (110) plane exhibited the lowest corrosion current density value of $0.009 \mathrm{~mA} / \mathrm{cm}^{2}$ and the corrosion potential value of $-0.380 \mathrm{~V}$, which showed the highest quality and corrosion resistance. Further, the first-principles calculation results demonstrated that for these coatings, a highest work function and lowest surface energy result in the weakest chemical activity, which provides a theoretical explanation for the corrosion resistance corresponding to the (110) plane. Based on the results of this work, a method is proposed to regulate $\mathrm{Cl}_{2}$ flow rate using consistent first-principles theory for the design and selection of Ta coatings that exhibit excellent resistance to anisotropic corrosion.

Keywords: chemical vapor deposition tantalum, selective corrosion, chlorine flow rate, first-principles calculation, simulated polarization curves.

\section{$\underline{\text { FULL TEXT }}$}

(C) 2022 The Authors. Published by ESG (www.electrochemsci.org). This article is an open access article distributed under the terms and conditions of the Creative Commons Attribution license (http://creativecommons.org/licenses/by/4.0/). 